\title{
MEDIDAS ESPIROMÉTRICAS EM PESSOAS EUTRÓFICAS E OBESAS NAS POSIÇÕES ORTOSTÁTICA, SENTADA E DEITADA
}

\author{
Nilsa Cristina Domingos-Benício, Ada Clarice Gastaldi*, José Cláudio Perecin, Kátia de Miranda Avena, \\ Rogério Contato Guimarães, Maria José Junho Sologuren, José Divino Lopes-Filho \\ Trabalho realizado no Centro Universitário do Triângulo (UNIT MG) e Universidade de Cuiabá (UNIC).
}

RESUMO - OBjetivo. Alguns autores demonstraram que a obesidade gera restrição pulmonar, levando a uma redução dos volumes pulmonares. Entretanto, ainda existem controvérsias acerca da existência dessa restrição e do possível mecanismo responsável por essa alteração. Frente a isso, esse estudo teve como objetivo avaliar a influência do excesso de peso corporal na espirometria, em três diferentes posiçóes corporais, avaliado pelo Índice de Massa Corpórea (IMC), percentual de gordura e relação cintura/quadril.

Métodos. Quarenta e seis universitários sedentários, com idades entre 20 e 40 anos, foram avaliados e distribuídos em cinco grupos, de acordo com o IMC. Os voluntários foram submetidos ao exame físico e à mensuração das pregas cutâneas e da circunferência da cintura e do quadril. CVF, VEF, e VEF/CVF foram avaliados em três diferentes posições - sentado, deitado e em pé.
Resultados. Quando comparados os valores obtidos e previstos entre os grupos, nenhuma diferença estatisticamente significante foi detectada. Quando comparadas as posições corporais, a posição deitada apresentou valores menores que as posições sentado e em pé $(p<0,05)$.Não foram observadas correlações entre os valores de CVF, VEF, e VEF/CVF e IMC, percentual de gordura e $\mathbf{R C} / \mathbf{Q}$.

ConcLusöes. Os valores espirométricos dos obesos estão dentro da faixa de normalidade e diminuem quando estes assumem a posição deitada.

Unitermos: Obesidade. Índice de Massa Corpórea. Função Pulmonar. Espirometria.

\section{INTRODUÇÃO}

A obesidade é um importante fator de risco para o desenvolvimento de disfunções orgânicas responsáveis pelo aumento do índice de morbidade e mortalidade e pela piora da qualidade de vida ${ }^{1,2}$. O avanço tecnológico atual, que induz a um estilo de vida sedentário, e fatores como o aumento do tabagismo, do consumo de álcool e do nível de estresse, vêm favorecendo o aumento da população obesa no País. É cada vez mais evidente a prevalência da obesidade e do sobrepeso nos diferentes segmentos da população brasileira ${ }^{3}$. Aproximadamente $32 \%$ da população adulta brasileira apresentam algum grau de sobrepeso $\left(\mathrm{IMC}>25 \mathrm{~kg} / \mathrm{m}^{2}\right)$, sendo que, destes, $8 \%$ apresentam obesidade $\left(I M C>30 \mathrm{~kg} / \mathrm{m}^{2}\right)^{4}$. Nos últimos 15 anos, houve um grande aumento da população de obesos no Brasil, sendo este mais significativo entre os homens4.

\footnotetext{
* Correspondência

Centro Universitário do Triângulo - UNIT MG Av. Nicomedes Alves dos Santos, 4545m - Gávea CEP: 38411-106 - Uberlândia - MG E-mail: ada@unitmg.com.br
}

Acredita-se que a obesidade gera restrição pulmonar devido à diminuição da excursão diafragmática pelo aumento da adiposidade abdominal ou do peso na parede torácica, levando a uma redução dos volumes pulmonares quando comparados com os valores previstos ${ }^{5}$. Entretanto, embora muitos estudos tenham sido realizados, ainda existem controvérsias sobre esta restrição e sobre qual seria o possível mecanismo responsável por essa alteração. Além disso, ainda não há definição acerca do comportamento do sistema respiratório de obesos nas diferentes posições corporais.

Alguns autores relatam diminuição na complacência total do sistema respiratório e na complacência da parede torácica ${ }^{6}$, enquanto outros sugerem que não existe correlaçãao entre a complacência da parede torácica e a obesidade ${ }^{7}$.

Em relação às posições corporais, alguns autores relataram aumento da capacidade vital forçada (CVF) e do volume expiratório forçado no primeiro segundo da expiração $\left(V E F_{1}\right)$ em indivíduos eutróficos na posição sentada quando comparada com a posição ortostática $^{8}$, enquanto outros autores não demonstraram diferença dos dados espirométricos $^{9}$ e complacência ${ }^{10}$ nas diferentes posições corporais. Entretanto, comparando indivíduos obesos e eutróficos na posição sentada e deitada, Yap et al." observaram, na posição sentada, redução da capacidade pulmonar total (CPT), da capacidade residual funcional (CRF), da CVF e do VEF, nos obesos, enquanto, na posição supina, houve aumento da CRF. Outro estudo demonstrou discreto aumento da CVF na posição em pé em relação à posição sentada'2.

Um estudo longitudinal com 507 indivíduos demonstrou uma redução da CV e do VEF, inversamente proporcional tanto ao aumento da prega subescapular quanto ao aumento da relação cintura/quadril (RC/Q). Entretanto, apenas a CVF apresentou associação negativa com o $\mathrm{IMC}^{13}$ e foram detectadas associações positivas entre o IMC e fluxo expiratório máximo e entre o IMC e a relação $\mathrm{VEF} / \mathrm{CVF}^{13}$. Collins et al. ${ }^{5}$, ao correlacionar os volumes pulmonares com a $R C / Q$, relataram diminuição na CVF, VEF, e CPT quando a RC/Q era maior que 0.95 , em indivíduos do sexo masculino. 
Outros estudos avaliaram a função pulmonar de indivíduos obesos sem comparar a posição corporal e encontraram diminuição do volume de reserva expiratório $(\text { VRE })^{14,15}$; discreta redução da CVF, capacidade inspiratória $(\mathrm{Cl})$ e CPT comparados aos valores previstos ${ }^{15}$.

Apesar do grande número de estudos realizados, ainda há controvérsias acerca da repercussão da obesidade sobre a função pulmonar e de identificar um parâmetro de avaliação da obesidade capaz de predizer estas repercussões. Frente a isso, o objetivo desse estudo foi avaliar a influência do excesso de peso corporal na função pulmonar, correlacionando IMC, percentual de gordura e relação cintura/quadril com os dados obtidos na espirometria, em três diferentes posições corporais.

\section{Métodos}

Foram avaliados 46 universitários sedentários (23 homens e 23 mulheres), provenientes da Universidade de Cuiabá (UNIC), com idades entre 20 e 40 anos, não-fumantes e sem história prévia de doenças cardiovasculares. Os voluntários obesos e com sobrepeso apresentaram-se espontaneamente e os eutróficos foram pareados por altura.

Inicialmente, os voluntários foram pesados e medidos em uma balança antropométrica - Fillizola, previamente calibrada pelo Inmetro, e, em seguida, foram distribuídos em cinco grupos, de acordo com o Índice de Massa Corpórea (IMC) e com base no Consenso Latinoamericano de Obesidade ${ }^{16}$. Os grupos apresentavam as seguintes características: GI (Peso Normal) - IMC $=20$ a $24,9 \mathrm{~kg} / \mathrm{m}^{2}$ (13 voluntários); G2 (Préobeso) - IMC = 25 a $29,9 \mathrm{~kg} / \mathrm{m}^{2}(14$ voluntários); G3 (Obesidade Grau I) IMC = 30 a $34,9 \mathrm{~kg} / \mathrm{m}^{2}$ (nove voluntários); G4 (Obesidade Grau II) - IMC = 35 a $39,9 \mathrm{~kg} / \mathrm{m}^{2}$ (dois voluntários); G5 (Obesidade Grau III) - IMC > $40 \mathrm{~kg} / \mathrm{m}^{2}$ (oito voluntários). Inicialmente, os voluntários foram submetidos ao exame físico para que se excluíssem doenças cardiopulmonares e a uma investigação, através de um questionário com a finalidade de avaliar tabagismo, tempo de obesidade e realização de atividade física. Em seguida, após um repouso de 15 minutos na posição supina em um ambiente climatizado a $27^{\circ} \mathrm{C}$, os voluntários foram submetidos à mensuração das pregas cutâneas e da circunferência da cintura e do quadril. Foram mensuradas as pregas subescapular, supra ilíaca e coxa nas mulheres; e as pregas triciptal, supra ilíaca e abdominal nos homens, obedecendo os procedimentos padronizados por Harrison ${ }^{17}$. Para a medida da circunferência cintura/ quadril, foi utilizada uma fita antropométrica flexível. Em seguida, foi realizado o exame espirométrico em três diferentes posições, (sentado, deitado e em pé), devidamente randomizadas e realizadas em três dias alternados. Os testes espirométricos foram realizados em um Espirômetro Vitatrace VT 130SL, utilizando um Sistema de Espirografia SSE - versão 3.2, conectado a um computador IBM e a uma impressora HP 680. Foram empregados os valores de referência propostos por Knudson et al. 18,19. Foram avaliados CVF, VEF, e VEF,/CVF. Todos os valores obtidos foram corrigidos e expressos em condições BTPS. Durante as mensurações, o voluntário foi continuamente estimulado através de um comando previamente estipulado. Os voluntários assinaram o consentimento pós-informado para participação em pesquisa. O estudo foi aprovado pelo Comitê de Ética em Pesquisa do Centro Universitário do Triângulo (UNIT MG).

Análise Estatística. Como os dados não acompanharam a distribuição normal, foram aplicados testes não paramétricos para a análise estatística. O teste de Friedman foi utilizado para comparar os valores de CVF, VEF, e VEF,/CVF entre voluntários eutróficos e obesos e para comparar esses valores nas posições sentado, deitado e em pé. Para as comparações entre os grupos, o grupo 4 não foi analisado devido ao pequeno número de voluntários $(n=2)$. A Correlação de Spearman foi utilizada para verificar existência de correlações entre as medidas de CVF, VEF, e VEF,/CVF com o IMC, o percentual de gordura e a relação cintura/ quadril. O nível de significância estatística foi estabelecido em 0,05.

\section{Resultados}

Os dados antropométricos, o percentual de gordura e a relação cintura/quadril dos voluntários dos grupos I a 5 estão descritos na Tabela I.

A análise dos resultados espirométricos, de acordo com a classificação estabelecida pela Americam Thoracic Society ${ }^{20}$, mostrou que os eutróficos (grupo I), com sobrepeso (grupo 2) e obesos (grupos 3, 4 e 5) apresentaram valores que, estatisticamente, não foram diferentes dos previstos. Analisando-se os resultados individuais, apenas duas pessoas apresentaram padrão restritivo na posição sentada (voluntários 2 e 8 , grupos 1 e 5 , respectivamente); enquanto três pessoas apresentaram padrão obstrutivo nas posições sentada e ortostática (voluntários I, 8 e II, grupos 5, 3 e 2, respectivamente).

Quando comparados os valores obtidos de CVF, VEF, e VEF, /CVF, não foi demonstrada nenhuma diferença estatisticamente significante entre os grupos.

Quando comparados os valores obtidos nas posições sentado e em pé, também não foram observadas diferenças significantes. Entretanto, na posição deitada, os valores obtidos foram menores que nas posições sentado e em pé $(p<0,05)$.

Os resultados de CVF, VEF, e VEF,/CVF obtidos nas posições sentado, deitado e em pé, para os cinco grupos, são apresentados nas Figuras I, 2 e 3, respectivamente.

O percentual de gordura, calculado a partir das medidas de pregas cutâneas, mostrou valores acima de $25 \%$ para o sexo masculino e acima de $33 \%$ para o sexo feminino, dos grupos 2 a 5 , o que caracteriza o aumento de peso por aumento de tecido gorduroso ${ }^{|6,2|}$.

De acordo com o teste de correlação de Spearman, não foram observadas correlações entre os valores obtidos de CVF e VEF, e o IMC, em nenhuma das três posições corporais. A relação $V E F$, CVF mostrou correlação com o IMC nas posições sentado e em pé.

Em relação aos outros parâmetros avaliados, também não foram encontradas correlações entre os valores de CVF e VEF e VEF,/CVF e percentual de gordura e RC/ $\mathrm{Q}$ quando analisados separadamente para homens e mulheres. Estes resultados estão demonstrados na Tabela 2. 


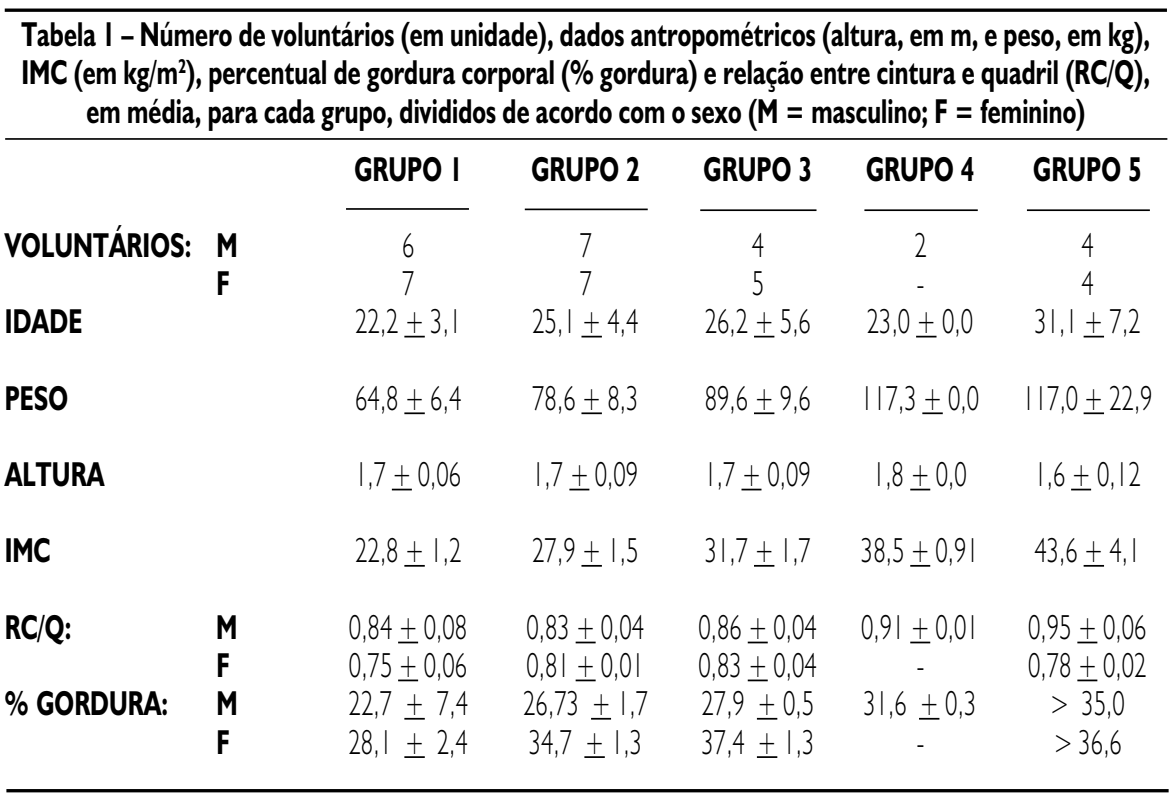

Figura I - Média e desvio padrão dos valores de capacidade vital forçada (CVF) para os grupos I a 5 nas posições sentada, deitada e ortostática

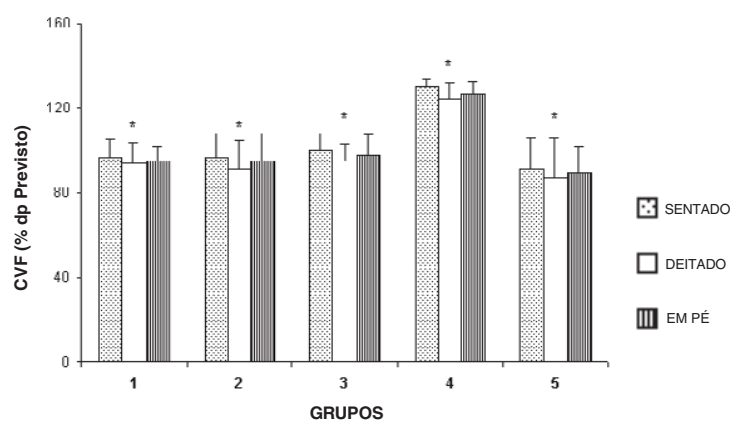

* $\mathrm{P}<0,05$ : valores deitado $<$ sentado e em pé

Figura 2 - Média e desvio padrão dos valores de volume expiratório forçado no primeiro segundo da expiração (VEF,) para os grupos I a 5 nas posiçōes sentada, deitada e ortostática

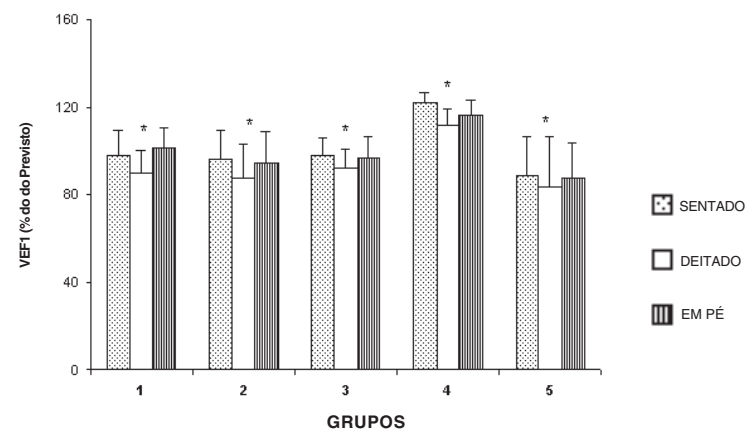

* $P<0,05$ : valores deitado < sentado e em pé

\section{Discussão}

A proposta deste estudo foi avaliar a função pulmonar, através da espirometria, de pessoas obesas, em diferentes graus de obesidade. Não foram encontradas diferenças entre os valores obtidos e previstos, para todos os grupos estudados.

Quando avaliados em diferentes posições, os valores de CVF foram menores na posição deitada e não foram diferentes nas posições sentado e em pé.

Existe na literatura um grande número de pesquisas que estudaram a restriçãa pulmonar gerada pela obesidade. Entretanto, as divergências acerca da existência dessa restrição e do seu mecanismo etiológico permanecem. Além disso, ainda existem controvérsias sobre o comportamento do sistema respiratório de obesos nas diferentes posições corporais, se 0 comportamento é semelhante aos indivíduos eutróficos ou não.

Avaliando grupos diversos de obesos, alguns autores encontraram diminuição de alguns volumes pulmonares, enquanto outros não encontraram alterações.

Nossos resultados estão de acordo com os de Gilbert, Siple \& Auchincloss ${ }^{14}$ e Kelly et al. ${ }^{22}$, que não encontraram alterações na CVF, VEF, $\mathrm{VEF} /$ / CVF e $\mathrm{FEF}_{25-75 \%}$ ao estudarem obesos classificados apenas pelo IMC.

Contrariamente, Ladosky, Botelho \& Albuquerque $\mathrm{R}^{23}$ constataram que os pacientes obesos apresentaram redução da ventilação voluntária máxima (WM), CVF, VEF VRE e pico de fluxo expiratório (PFE), inversamente proporcional ao aumento do IMC; e aumento da relação entre PFE e pico de fluxo inspiratório (PFI) - PFE/PFI, diretamente proporcional ao aumento do IMC.

Apesar de apresentarem volumes pulmonares dentro da normalidade, alguns estudos relatam que em indivíduos obesos, após a perda de peso, ocorre melhora dos volumes pulmonares ${ }^{24,25,26}$.

Até o presente momento, nenhum autor estabeleceu uma relação linear entre a função pulmonar e o aumento do peso corporal, entretanto, alguns estudos mostraram que a perda de peso melhora a função pulmonar. Bedell, Wilson \& Seebohm ${ }^{24}$, Crapo et al.,$^{25}$ e Thomas et al. ${ }^{26}$ demonstraram aumento de todos os volumes pulmonares com a perda de peso, 


\section{Figura 3 - Média e desvio padrão dos valores da relação entre volume expiratório forçado no primeiro segundo da expiração (VEF) e capacidade vital forçada (CVF), VEF,CVF, para os grupos I a 5 nas posições sentada, deitada e ortostática}

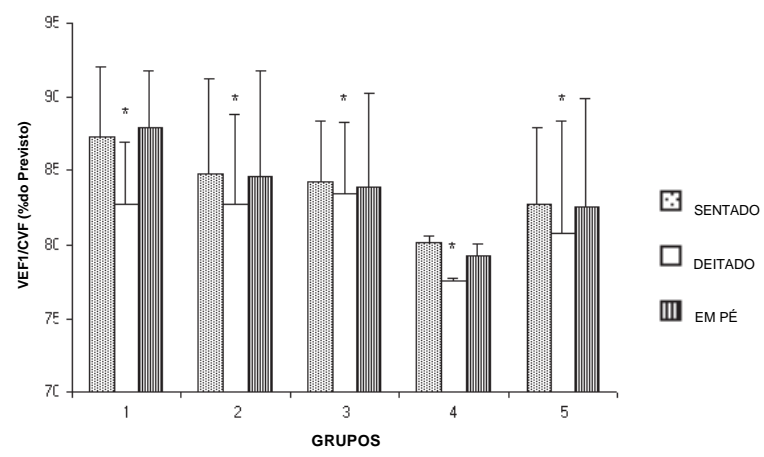

* $P<0,05$ : valores deitado $<$ sentado e em pé

\begin{tabular}{|c|c|c|c|c|c|c|c|}
\hline \multicolumn{8}{|c|}{$\begin{array}{l}\text { Tabela } 2 \text { - Correlações entre as medidas espirométricas e o Índice de Massa } \\
\text { Corpórea (IMC), o percentual de gordura corporal (\% Gordura) e a relação entre cintura } \\
\text { e quadril ( }(\mathrm{CC} / \mathrm{Q} \text { ) nas posiçōes sentado, deitado e em pé }\end{array}$} \\
\hline & & \multicolumn{2}{|c|}{ CVF } & \multicolumn{2}{|c|}{ VEFI } & \multicolumn{2}{|c|}{ VEFI/CVF } \\
\hline & & $r$ & $p$ & $r$ & $p$ & $r$ & $p$ \\
\hline SENTADO & $\begin{array}{l}\text { IMC } \\
\mathrm{RC} / \mathrm{Q} \\
\% \text { Gordura } \\
\text { IMC }\end{array}$ & $\begin{array}{r}0,10 \\
0,04 \\
-0,06 \\
0,06\end{array}$ & $\begin{array}{l}0,50 \\
0,80 \\
0,69 \\
0,71\end{array}$ & $\begin{array}{l}0,07 \\
-0,07 \\
-0,05 \\
0,09\end{array}$ & $\begin{array}{l}0,60 \\
0,60 \\
0,76 \\
0,57\end{array}$ & $\begin{array}{l}-0,35 \\
-0,38 \\
-0,07 \\
-0,04\end{array}$ & $\begin{array}{l}0,02 \\
0,01 \\
0,64 \\
0,80\end{array}$ \\
\hline DEITADO & $\begin{array}{l}\mathrm{RC} / \mathrm{Q} \\
\text { \% Gordura } \\
\text { IMC } \\
\mathrm{RC} / \mathrm{Q} \\
\text { \% Gordura }\end{array}$ & $\begin{array}{l}0,03 \\
-0,07 \\
-0,12 \\
0,04 \\
-0,21\end{array}$ & $\begin{array}{l}0,80 \\
0,64 \\
0,43 \\
0,80 \\
0,16\end{array}$ & $\begin{array}{l}0,23 \\
-0,02 \\
-0,18 \\
0,04 \\
-0,20\end{array}$ & $\begin{array}{l}0,80 \\
0,88 \\
0,23 \\
0,8 \\
0,17\end{array}$ & $\begin{array}{l}-0,23 \\
0,17 \\
-0,32 \\
-0,17 \\
-0,06\end{array}$ & $\begin{array}{l}0,12 \\
0,24 \\
0,03 \\
0,26 \\
0,71\end{array}$ \\
\hline
\end{tabular}

enquanto Vaughan, Cork \& Hollander ${ }^{27}$ demonstraram melhora apenas do VRE, que se correlacionava diretamente com 0 aumento da pressão parcial de oxigênio no sangue arterial $\left(\mathrm{PaO}_{2}\right)$ e da diferença artério-venosa de oxigênio $\left(\mathrm{Ca}-\mathrm{vO}_{2}\right)$. Esses dados sugerem a possibilidade de uma adaptação progressiva do sistema respiratório frente ao aumento de peso a longo prazo. Em um recente estudo, Hakala, Stenius-Aarniala \& Sovijarvii ${ }^{28}$, após avaliarem pacientes obesos submetidos à dieta durante oito semanas, verificaram que houve, após a perda de peso, aumento do PFE, CVF, VEF $, \mathrm{FEF}_{25-75 \%}, \mathrm{FEF}_{25-75 \%} / \mathrm{CVF}$, CRF e CPT; enquanto que não houve modificação no VEF, /CVF, VR e VR/CPT. Os voluntários obesos selecionados para este presente estudo eram obesos há mais de um ano. Portanto, é possível sugerir que, por apresentarem obesidade a longo prazo, tenham desenvolvido mecanismos adaptativos contra a sobrecarga imposta pelo tecido adiposo e, por isso, não apresentam reduções importantes dos valores

Apesar de não ser um indicador específico para gordura, o IMC é recomendado para uma primeira classificação de população, que, posteriormente, deve ser submetida a avaliações sucessivamente mais refinadas, como percentual e distribuição da gordura corporal, porém é importante ressaltar que a grande maioria dos estudos realizados considera apenas o IMC como referência para a classificação de obesidade.

Embora esse índice seja recomendado pelo Consenso Latino-americano de Obesidade ${ }^{16}$ como o primeiro referencial para essa classificação, o IMC isoladamente não espirométricos. pode ser considerado como um bom indicador por ser incapaz de diferenciar osso, músculo e gordura ${ }^{3,29}$.

Rubinstein et al. ${ }^{30}$ afirmam que existe correlação entre $I M C$ e a função pulmonar, exceto para os valores de VEF,/CVF. Fung et al. ${ }^{31}$, após estudar adolescentes de ambos os sexos e garotas com sobrepeso, confirmaram os resultados acima descritos. Lazarus et al.13 descreveram uma relação inversa entre CVF e IMC; uma relação direta entre VEF,/CVF e IMC; e não encontraram correlações entre VEF e IMC. Ladosky et al. ${ }^{23}$, ao compararem pacientes eutróficos e obesos, relataram redução dos valores de CVF e VEF, proporcional ao aumento do IMC. Nossos resultados não mostraram correlação entre IMC e CVF e VEF. Nas posições sentada e em pé, apenas a relação VEF,/CVF mostrou correlaçãa negativa. Porém a observação desta correlação dentro de um grupo com CVF, VEF, e relação $V E F, /$ CVF dentro da normalidade, este dado não sugere uma implicação clínica.

O parâmetro recomendado pelo Consenso Latino-americano de Obesidade ${ }^{16}$ para avaliação da distribuição de gordura é a relação da cintura/quadril (RC/Q). De acordo com Collins et al. ${ }^{5}$, seria esperado um comportamento inversamente proporcional entre $\mathrm{RC} / \mathrm{Q}$ e os volumes pulmonares, tendo em vista que uma RC/Q aumentada caracteriza a distribuição de gordura na região abdominal e torácica, produzindo maior resistência na parede do tórax e, com isso, reduzindo a complacência e os volumes pulmonares. Entretanto, o presente estudo não constatou correlação entre RC/Q e os valores obtidos na espirometria, confirmando os achados de Lazarus et al..$^{29}$.

A ausência de correlação entre RC/Q e os valores espirométricos pode ser justificada pela teoria de que, nas pessoas obesas, à medida que ocorre redução do VRE, ocorre o aumento da capacidade inspiratória, e, talvez, a diminuição dos valores espirométricos ocorra apenas quando não há mais reservas metabólicas suficientes para sustentar o alto consumo energético responsável pela manutenção do trabalho respiratório aumentado ${ }^{22}$.

O provável mecanismo para a restriçãa pulmonar seria devido ao efeito do conteúdo abdominal sobre a posição do diafragma, com comprometimento do volume de reserva expiratório e diminuição da ventilação das 
bases pulmonares. Entretanto, em alguns estudos, a força muscular diafragmática é normal e não há comprometimento da complacência da parede torácica.

Apesar das questões ainda existentes sobre a possível alteração pulmonar, obstrução ou restrição, e de os achados deste presente estudo mostrarem valores de CVF dentro da normalidade, vale ressaltar que em alguns casos isolados houve restrição ou obstrução pulmonar. Isto sugere que os resultados devem ser analisados cautelosamente.

Reforçando esta hipótese, em recente estudo, Sin et al..$^{32}$ encontraram maior risco de relatos de asma, uso de broncodilatadores e relatos de dispnéia entre grupos de obesos, porém os voluntários com maiores IMC tiveram menor freqüência de obstrução ao fluxo aéreo.

Essas controvérsias podem ser devidas aos métodos utilizados. É importante que futuras pesquisas estudem, isoladamente, diferentes graus de obesidade; que a seleção de voluntários e seqüência de procedimentos seja aleatória; e que sejam utilizados diversos métodos para classificação dos obesos.

Outra questão importante é a diminuição de volumes quando passam da posição sentada para deitada. Os obesos, de modo semelhante ao que ocorre em eutróficos, perdem cerca de $20 \%$ de sua CVF ao assumirem a posição deitada. Isso tem sérias implicações clínicas, especialmente se associado a alterações pulmonares prévias, exigindo especial atenção para aqueles obesos que permanecem restritos ao leito por longos períodos de tempo.

\section{Conclusões}

Os resultados encontrados sugerem que não há diferença entre os valores de CVF, VEF, VEF,/CVF em indivíduos eutróficos e obesos, mesmo para o grupo de obesos com IMC > $40 \mathrm{~kg} / \mathrm{m}^{2}$; e que há uma redução do CVF, VEF, VEF,/CVF na posição deitada, tanto para indivíduos eutróficos quanto para indivíduos obesos. Não é possível afirmar que exista uma relação direta entre os valores da espirometria e 0 IMC, percentual de gordura e $R C / Q$, quando correlacionados isoladamente.

Conflito de interesse: não há.

\section{SUMMARY}

\section{Spirometric values of obese and NON-OBESE SUBJECTS ON ORTHOSTATIC, SITTING AND SUPINE POSITIONS}

BACKGROUND. It is possible that obesity could lead to pulmonary restriction with decreasing lungvolumes. However, controversies about this restriction and its etiology still exist. Thus, the purpose of this report was to evaluate the effects of body weigh excess on spirometry, on three different body positions, evaluated by Body Mass Index (BMI), percentage of fatness and the ratio of abdominal girth to hip breadth (AG/HB).

METHODS. Forty-six sedentary volunteers, with ages between 20 and 40 years, were studied and distributed on five groups, based on BMI. Skin fold thickness and ratio of abdominal girth to hip breadth $(A G / H B)$ of the volunteers were measured. FVC, FEV and ratio of FEV , to FVC were measured on three different body positions - sitting, supine and orthostatic positions.

RESULTS. Comparing the values measured and predicted between the groups, no difference was detected. Comparing body positions, the supine position shows lower values than sitting and orthostatic positions $(p<0.05)$. Associations between CVF, VEF e VEF/CVF values and $B M I$, percentage of fatness and ratio of $A G / H B$ werenotfound.

Conclusions. Spirometric values from obese people are into normality range and decrease on the supine position. [Rev Assoc Med Bras 2004; 50(2): 142-7]

KEY WORDS: Obesity. Body Mass Index. Pulmonary Function. Spirometry.

\section{REFERÊNCIAS}

I. James WPT. A public health approach to the problem of obesity. Int J Obesity 1995; 19(Suppl 3):37-45

2. Gibson GJ. Obesity, respiratory function and breathlessness. Thorax 2000; 55 (Suppl 1): $54 \mid-4$.

3. Guedes DP, Guedes JERP. Controle do peso: composição corporal, atividade física e nutrição. Londrina: Midiograf; 1998.

4. INAN. Condições Nutricionais da População Brasileira: adultos e idosos. Pesquisa nacional sobre saúde e nutrição. Brasília: Instituto Nacional de Alimentação e Nutrição, Ministério da Saúde; |99|.

5. Collins CL, Hoberty PD, Walker JF, Fletcher EC, Peiris AN. The effects of body fat distribution on pulmonary function tests. Chest 1995; 107:1298-302.
6. Naimark A, Cherniack RM. Compliance of the respiratory system and its components in health and obesity. J Appl Physiol 1960, 15:377-82.

7. Surrat PM, Wilhoit SC, Hsiao HS, Atkinson RL, Rochester DF. Compliance of chest wall in obese subjects. J Appl Physiol I 984, 57:403-7.

8. Pierson DJ, Dick NP, Petty TL. A comparison of spirometric values with subjects in standing and sitting positions. Chest 1976; 70: 17-20.

9. Towsend MC. Spirometric forced expiratory volumes measured in the standing versus sitting posture. Am Rev Respir Dis 1984; 130:123-4

10. Burki NK. The effects of posture on total respiratory compliance. Am Rev Respir Dis 1982; 125:262-3.

II. Yap JCH, Watson RA, Gilbey S, Pride NB. Effects of posture on respiratory mechanics in obesity. J Appl Physiol 1995, 79:1 199-205.

12. Gudmundsson G, Cerventy M, Shasby M. Spirometric values in obese individuals: effects of bodypositions. Am J Respir Crit Care Med 1997; । 55:998-9.

13. Lazarus R, Sparrow D, Weiss ST. Effects of obesity and fat distribution on ventilatory function-the normative aging study. Chest 1997; | | |:89|-8.

14. Gilbert R, Siple JH, Auchincloss JH. Respiratory control and work of breathing in obese subjects. J Appl Physiol 1961, 16:21-6.

15. Barrera F, Reidenberg MM, Winters WL. Pulmonary function in the obese patient. Am J Med Sci 1967; 254:785-96.

16. Consenso Latino-americano de Obesidade. Arq Bras Endocrinol Metabol 1999; 43(I).

17. Harrison GG. Skinfold thickness and measurement technique. In: Lohman TG, Roche AF, Martorell M. Anthropometric standardization reference manual. Champaign: Human Kinetics Books; 1998. p.55-80.

18. Knudson RJ, Slatin RC, Lebowitz MD, Burrows B. The maximal expiratory flow-volume curve: normal standards, variability and effects of age. Am Rev Respir Dis 1976; 1 13:587.

19. Knudson RJ, Lebowitz MD, Holberg CJ, Burrows B. Changes in the normal maximal expiratory flow-volume curve with growth and aging. Am Rev Respir Dis 1983; 1 27:725-34.

20. American Thoracic Society. Standardization of spirometry. Am J Respir Crit Care Med 1995; | 52: | 1 07-36.

21. Heyward VH, Stolarczyk LM. Método de dobras cutâneas. In: Heyward VH, Stolarczyk LM, editores. Avaliação da composição corporal aplicada. Rio de Janeiro: Manole; 2000. p.23-46.

22. Kelly TM, Jensen RL, Elliott CG, Crapo RO. Maximun respiratory pressures in morbidly obese subjects. Respiration 1988; 54:73-7.

23. Ladosky W, Botelho MAM, Albuquerque Jr JP. Chest mechanics in morbidly obese nonhypoventilated patients. Respir Med 200I; 94:28I-6.

24. Bedell GN, Wilson WR, Seebohm PM. Pulmonary function in obese persons. I Clin Invest 1958; 37: 1049-60. 
25. Crapo RO, Kelly TM, Elliott CG, Jones SB. Spirometry as a preoperative screening test in morbidly obese patients. Surgery 1986; 99:763-7.

26. Thomas PS, Owen ERTC, Hulands G, Milledge JS. Respiratory function in the morbidly obese before and after weight loss. Thorax 1989; 44:382-6.

27. Vaughan RW, Cork RC, Hollander D. The effects of massive weight loss on arterial oxygenation and pulmonary function tests. Anesthesiology 1981; 54:325-8.
28. Hakala K, Stenius-Aarniala B, Sovijarvi A. Effects of weight loss on peak flow variability, airways obstruction and lung volumes in obese patients with asthma. Chest 2000; | 18:1315-21.

29. Lazarus R, Gore C], Booth M, Owen N. Effects of body compositions and fat distribution on ventilatory function in adults. Am J Clin Nutr | 998; 68:35-41.

30. Rubinstein I, Zamel N, Dubarry L, Hoffstein $\checkmark$. Airflow limitation in morbidly obese nonsmoking men. Ann Intern Med 1990; I 12:828-32.
31. Fung KP, Lau SP, Chow OKW, Lee J, Wong TW. Effects of overweight on lung function. Arch Dis Child 1990; 65:512-5.

32. Sin DD, Jones RL, Man P. Obesity is a risk factor for dyspnea but not for airflow obstruction.

Artigo recebido: 19/12/2002

Aceito para publicação: 26/I I/2003

\section{www.ramb.org.br}

A Ramb disponibiliza agora um novo serviço para os nossos colaboradores: 0 envio de artigos pela internet. Através do nosso publicador, que agiliza o fluxo de submissão com mais rapidez e versatilidade, você poderá enviar seu artigo para publicação na Ramb.

Experimente: é muito mais cômodo, muito mais rápido e você ainda poderá acompanhar on line o curso de sua apreciação. 